\title{
A Capacitated Facility Location Problem with Constrained Backlogging Probabilities
}

\author{
Francisco José Ferreira Silva (corresponding author) \\ GREL, IET, Universitat Pompeu Fabra \\ Ramon Trias Fargas, 25-27, 08005 Barcelona, Spain \\ CEEAplA, Universidade dos Açores \\ Rua da Mãe de Deus, 9502 Ponta Delgada, Portugal \\ Telephone: (+351) 296650555 \\ Fax: (+351) 296650083 \\ email: francisco.silva@upf.edu
}

\section{Daniel Serra}

GREL, IET, Universitat Pompeu Fabra

Ramon Trias Fargas, 25-27, 08005 Barcelona, Spain

Telephone: (+34) 935421666

Fax: (+34) 935421746

email: daniel.serra@upf.edu 


\begin{abstract}
One of the assumptions of the Capacitated Facility Location Problem (CFLP) is that demand is known and fixed. Most often, this is not the case when managers take some strategic decisions such as locating facilities and assigning demand points to those facilities. In this paper we consider demand as stochastic and we model each of the facilities as an independent queue. Stochastic models of manufacturing systems and deterministic location models are put together in order to obtain a formula for the backlogging probability at a potential facility location.

Several solution techniques have been proposed to solve the CFLP. One of the most recently proposed heuristics, a Reactive Greedy Adaptive Search Procedure, is implemented in order to solve the model formulated. We present some computational experiments in order to evaluate the heuristics' performance and to illustrate the use of this new formulation for the CFLP. The paper finishes with a simple simulation exercise.
\end{abstract}

Keywords: Location, queuing, greedy heuristics, simulation.

JEL: C61, L80 


\section{Introduction}

Transportation costs and location-specific fixed costs are often a major component of the price (cost) of goods. The Facility Location Problem (FLP), introduced by (Balinski 1965) addresses the problem of locating a new set of facilities in such a way that the sum of those two costs is minimized.

Another concern when designing and operating a manufacturing system is the capacity the system tolerates: given the processing facilities, what is the maximum rate of order receipt that can be accepted so that all the orders can be satisfied? The Capacitated Facility Location Problem (CFLP) is a variant of the FLP, which includes capacities for the facilities. With the inclusion of the capacities, an open facility that is the least cost source for a demand node may not be able to serve any of the demand at that node.

The capacities of the facilities as well as the demand at each of the demand nodes have been assumed to be known deterministic parameters. In this paper we relax these assumptions by considering that the demand is stochastic following a given probability distribution and where capacity at each facility results from the probability of losing or backlogging the demand.

Stochastic models on manufacturing systems give us some important results, using analytical techniques such as stochastic processes, queuing theory and reliability theory, which allow the computation of the referred probabilities as a function of arrival and service rates. In this paper we introduce these considerations in the CFLP. The objective is to find the best location of facilities (the one that minimizes total transportation and fixed costs) maintaining the probability of losing /backlogging demand on a small level. 
The CFLP considers that distinct potential facility sites present different fixed costs for locating a facility, that facilities being sited are constrained to a given capacity level on the demand they can serve and that we do not know, a priori, the optimal number of facilities to be opened. These assumptions make from the CFLP a complex problem that is difficult to solve. There is a vast literature concerning the development and testing of new algorithms that search for the solution to the problem.

The most common approach to solving the CFLP is the use of Lagrangean heuristics. These heuristics are based on a Lagrangean relaxation and some method for solving the Lagrangean dual problem. More recently Greedy Heuristics, Tabu Search and Genetic Algorithms have been proposed to solve the CFLP. Based on previous research we will propose a heuristic algorithm to solve the new version of the model.

The paper is organized as follows: in section 2 we describe the Single Source Capacitated Facility Location Problem; in section 3 we give a brief description of stochastic manufacturing models whose results are to be used in section 4 in order to formulate the Queue Length Capacitated Facility Location Problem. In section 5 we describe heuristics to solve the problem and finally in section 6 we offer some numerical examples.

The motivation for the paper results from the fact that this model may allow a rapid analysis of many manufacturing alternatives enabling the firm to take rapid decisions both in the design and in the operation phases, and to obtain some competitive advantages in costs resulting from vantages on the stock management policy.

\section{The Single Source Capacitated Facility Location Problem (SSCFLP)}

Facility Location Problems (FLP) deserved a special place in Location Literature in the second half of last century. Some important summaries of the state of the art can 
be found in (Balinski and Spielberg, 1969), (ReVelle et al., 1970), (Guignard and Spielberg, 1977), (Cornuejols, 1978) or (Krarup and Pruzan, 1983).

The FLP derives its name from the analogy to decision problems concerning the location of plants or facilities (e.g. factories, warehouses, schools) so as to minimize the total cost of serving clients (e.g. depots, retail outlets, students). (Krarup and Pruzan, 1983) refer their own experience as consultants where they have utilized FLP formulations as the basis for providing decision inputs to real-world problems regarding the number, size, design, location, and service patterns for such widely varied 'plants' as high-schools, hospitals, silos, slaughterhouses, electronic components, warehouses, as well as traditional production plants. As referred by the same authors the FLP permits in a sense the broadest framework. Neither the number of plants to be located nor the transportation or communication pattern is predetermined. Furthermore, the basic formulation of FLP lends itself readily to sensitivity analyses. In addition, FLP invites modifications which may permit more 'realistic' modeling. While FLP is basically a discrete, static, deterministic, one-product, fixed-plus-linear costs minimization problem formulation, it can be modified to accommodate dynamic, stochastic, multi-product, nonlinear cost minimization formulations.

The first explicit formulation of FLP is frequently attributed to (Balinski, 1966) whose expository article on integer programming includes the mixed-integer formulation. The paper was presented at the IBM Scientific Symposium on Combinatorial Problems in March 1964 but remained unpublished until 1966. However, FLP's are also dealt with in the pioneering papers by (Kuehn and Hamburger, 1963) and (Manne, 1964). 
FLP, Plant Location Problems consider situations in which a commodity is supplied from a subset of plants, selected from a set of potential location sites, to satisfy the demand of a set of clients. There are fixed costs for opening the plants and transportation costs to supply the commodity or the standard product-mix from potential location sites to clients. The decision maker seeks for a combination of minimum costs in terms of the plants to be opened and the allocation of clients within the subset of open plants.

The simplest formulation of FLP is the Uncapacitated Facility Location Problem (UFLP). It considers that the plants have unlimited capacity. There are several application for the UFLP, for example, bank account location (Cornuejols et al., 1977), economic lot sizing (Krarup and Blide, 1977), machine scheduling (Hansen and Kaufman) or portfolio management (Beck and Mulvey).

Let $I=\{1, \ldots, m\}$ be a set of customers which are to be served from plants located in a subset of sites from a given set $J=\{1, \ldots, n\}$ of potential sites. For each site $\mathbf{j} \in \mathbf{J}$, the fixed cost of opening the plant at $j$ is $\mathrm{f}_{\mathrm{j}}$. The cost of assigning site $\mathrm{j}$ to customer $\mathrm{i}$ is $c_{i j}$. Considering, $X_{i j}=\left\{\begin{array}{rr}1 & \text { if facility j serves } \text { customer i } \\ 0 & \text { otherwise }\end{array}\right.$ $Y_{j}=\left\{\begin{array}{lr}1 & \text { if facility } \mathrm{j} \text { is opened } \\ 0 & \text { otherwise }\end{array}\right.$ the model can be formulated as follows: 
$\min \sum_{i=1}^{m} \sum_{j=1}^{n} c_{i j} X_{i j}+\sum_{j=1}^{n} f_{j} Y_{j}$

s.t.

$\sum_{j=1}^{n} X_{i j}=1 \quad \forall i$

$\begin{array}{ll}X_{i j}-Y_{j} \leq 0 & \forall i, \forall j \\ X_{i j} \in\{0,1\} & \forall i, \forall j \\ Y_{j} \in\{0,1\} & \forall j\end{array}$

The CFLP considers a situation in which the plants have a capacity expressed in units of demand and also assumes that each client can be served from different open plants. Several applications for CFLP have been studied in literature: optimal lot sizing decisions in production planning ( Krarup and Blide), telecommunications network design ( Kochman and McCallum).

Let $a_{i}, i \in I$, be the customer's demands and $b_{j}$ the facility's capacity, then the CFLP is formulated as:

$$
\begin{array}{lc}
\min \sum_{i=1}^{m} \sum_{j=1}^{n} c_{i j} X_{i j}+\sum_{j=1}^{n} f_{j} Y_{j} \\
\text { S.t. } & \forall i \\
\sum_{j=1}^{n} X_{i j}=1 & \forall i, \forall j \\
\sum_{i=1}^{m} a_{i} X_{i j} \leq b_{j} Y_{j} & \forall i, \forall j \\
0 \leq X_{i j} \leq 1 & \forall j \\
Y_{j} \in\{0,1\} &
\end{array}
$$

When an additional restriction that forces each customer to be served only from a single facility is added we obtain the Single Source Capacitated Facility Location Problem (SSCFLP). The single source issue is typical for real life situations where multiple deliveries may increase the cost of maintaining and updating the inventory. 
This problem is in general more difficult to solve because the decision variables are binary. Another assumption of the SSCFLP considers that transportation costs from facilities to markets are linear according to the quantity transported (i.e., there are no economies of scale and the production costs at a facility are linear in the quantity produced once an initial fixed cost has been incurred). This problem has been widely studied in the literature, and for review purposes, see as an example Sridharan (1995).

$$
\begin{array}{ll}
\min \sum_{i=1}^{m} \sum_{j=1}^{n} c_{i j} X_{i j}+\sum_{j=1}^{n} f_{j} Y_{j} \\
\begin{array}{ll}
\text { S.t. } \\
\sum_{i=1}^{m} a_{i} X_{i j} \leq b_{j}
\end{array} \\
\sum_{j=1}^{n} X_{i j}=1 & \forall j \\
X_{i j}-Y_{j} \leq 0 & \forall i, \forall j \\
X_{i j} \in\{0,1\} & \forall i, \forall j \\
Y_{j} \in\{0,1\} & \forall j
\end{array}
$$

The objective function minimizes the cost of assigning customers to open facilities and the cost of establishing such facilities. Constraint set 2.9 can be referred to as the capacity constraints (or the facility constraint), and ensures that the customer demand served by a certain facility does not exceed its capacity. Constraint set 2.10 can be referred to as the demand constraints (or the customer constraints), and ensures that each customer is assigned to exactly one facility. Finally, constraint set 2.11 ensures that the assignments are made only to open facilities. In this model all decision variables are binary.

Constraint set 2.9 and constraint set 2.11 may be concentrated in the following constraint: 


$$
\sum_{i=1}^{m} a_{i} X_{i j} \leq b_{j} Y_{j} \quad \forall j
$$

Nevertheless, in order to facilitate the formulation of the new model we will keep the initial configuration.

\section{Stochastic Models for Manufacturing Systems}

Stochastic models for manufacturing systems have been developed for more than half a century. These models were developed as an attempt to provide analytical formulas that would predict the performance of manufacturing systems. For a good review see (Suri et al. 1993). Models which explicitly make use of queuing theory were first developed to solve machine interference problems. Interference problems result from the non-synchronized use of the machines and are concretized when down machines are interfering with operating ones. Queuing theory is the most common methodology for solving this type of problems. For a good review on early models, see (Stecke et al. 1985), and for a detailed mathematical description of the models and their applications see (Buzacott et al. 1993).

The two traditional forms of organizing manufacturing systems are the job shop and the flow lines. The main difference between the two forms consists of the fact that the flow lines system requires all jobs to visit all machines and work centres in the same sequence which is not the case in the job shops, where we may alter the sequence. Job shops obey to two different configurations: produce-to-order, where the job order arrives from outside the shop (stocks are not allowed) and produce-to-stock, where the job orders will be influenced by the stock levels. In this paper we are concerned with produce-to-stock systems. Produce-to-stock operations should reduce the delay in filling customer's orders and may lead to increased sales. The cost of keeping inventories is 
also expected to be higher with this system leading to the need for careful stock management.

'System design' is the term used to specify the rules that determine how production authorizations are generated. In this paper we restrict the discussion to single stage manufacturing facilities. Completed items of each product are kept in an output store. As customers arrive, their demands are met by delivering to them items from the output store. If all demands cannot be met immediately, two alternatives will be considered: lost sales and back-logged demand (where the customer waits until his required demand is met).

Now, consider the well known Production Authorization (PA) Cards System. In a simple formula the system works as follows: each item produced by the manufacturing facility has a tag associated, and when an item of a given product is delivered to a customer the tag is removed and becomes a production authorization or PA card for that product. The PA card can be directed to the production facility as soon as it is generated or wait until a batch of PA cards accumulates. The notation used in this paper is quite close to the one used by (Buzacott et al. 1993). For a complete description of the models or to find out about other models on the same line of research refer to this textbook.

For the purposes of this paper we will consider a single stage manufacturing system that produces items of a single product type to stock. Completed items are kept in a store from which customer demands are met. Customers arrive according to a process $\left\{A_{n}, n=1,2, \ldots\right\}, A_{n}$ is the arrival time of the $n t h$ customer. Let us assume that each customer asks for only one unit of the product. If a customer's demand cannot be met from available stock, the customer will wait until his demand can be satisfied. The manufacturing process of items involves the transformation of the raw material by 
processing it on a single machine. Items are processed one at a time and the processing time of the $n$th item is $S_{n}, n=1,2 \ldots$

Consider the PA mechanism that stops production as soon as the number of items in store reaches a target level, say $\mathrm{Z}$ (we will denote $\mathrm{Z}$ as the capacity level). Production authorization, in this case, is transmitted to the manufacturing facility only when the number of completed items is fewer than this target. Additionally, let us assume that there is a single production unit to process the items. When there are $r$ finished items in the output store, $r$ of these tags are attached, one for each of the finished items. The remaining Z-r tags will be available at the machine acting as PA cards. Consider the additional assumption that the store is full at time zero.

Let $I(t)$ be the inventory, that is, the number of finished items, in the output store, $R(t)$ be the number of items delivered to customers, $B(t)$ be the number of customers backlogged, and $C(t)$ be the number of PA cards available at the machine at time t. Then

$$
\begin{aligned}
& I(t)=Z-C(t)=Z+D(t)-R(t) \\
& R(t)=\min \{Z+D(t), A(t)\} \\
& B(t)=A(t)-R(t) \\
& C(t)=\min \{A(t)-D(t), Z\}
\end{aligned}
$$

where, $A(t)$ is the number of customers that arrived during $(0, t]$ and $\mathrm{D}(\mathrm{t})$ is the number of items produced during $(0, t]$.

Equation (8) states that the inventory equals the number of tags not available, i.e. the total number of tags $(\mathrm{Z})$ minus the number of available tags (resulting from the products sold during $(0, t]$ and not yet replenished by products produced in the same period of time). Equation (9) tells us that the number of sold items will be equal to demand whenever there is a sufficient number of items to meet this demand (initial stock plus production). The number of customers backlogged equals demand minus 
effective sales (equation 10) and the number of available PA cards equals demand minus production, with an upper bound of $\mathrm{Z}$ (equation 11).

Let $N(t)$ be the number of jobs in the single server queuing system described earlier. Then,

$$
N(t)=A(t)-D(t)
$$

i.e., the number of jobs in the system is equivalent to the number of tags that became available in $(0, t]$ minus the ones that were attached to new items produced in this time period.

Subtracting equation (10) from equation (8) results in the following expression

$$
I(t)-B(t)=Z+D(t)-A(t)=Z-N(t)
$$

$I(t)>0$ implies $B(t)=0$ (i.e. whenever there is a positive inventory backlogging is zero) and $B(t)>0$ implies $I(t)=0$ (we have backlogging when inventory is zero).

Using result (13) we have that

$$
I(t)=\{Z-N(t)\}^{+}
$$

and

$$
B(t)=\{N(t)-Z\}^{+}
$$

Assuming an M/M/1 model where the customer arrival process is Poisson with rate $\lambda$ and the processing times are exponentially distributed with mean $\frac{1}{\mu}$, then we have, from queuing theory that

$$
\begin{aligned}
& P\{N=n\}=(1-\rho) \rho^{n} \\
& \text { and } \\
& P\{N \leq n\}=1-\rho^{n+1}
\end{aligned}
$$

Backlogging probability will be the same as the probability of having zero inventories, and can be computed as 


$$
P\{I=0\}=P\{B(t)>0\}=P\{N(t)>Z\}=1-P\{N(t) \leq Z-1\}=\rho^{Z}
$$

We may also also consider a single facility produce-to-stock system in which customer demands not met by items of the output store are lost. Again, assuming thet customers arrive according to a Poisson process with rate $\lambda$ which is independent of the sequence of processing times and that the processing times are iid with exponential distribution with mean $\frac{1}{\mu}$, then in this case $\mathrm{N}(\mathrm{t})$ is a birth-death process on the state space $S=\{0, \ldots, Z\}$. Solving the flow balance equations one obtains:

$$
P\{N=n\}=\frac{(1-\rho) \rho^{n}}{1-\rho^{Z+1}} \quad \text { for } \mathrm{n}=0, \ldots, \mathrm{Z}
$$

and the probability of lost sales (inventory is zero)

$$
P\{I=0\}=\frac{(1-\rho) \rho^{Z}}{1-\rho^{Z+1}} \quad \text { for } \mathrm{n}=0, \ldots, \mathrm{Z}
$$

Given that this last expression is numerically more difficult to work when comparing with expression (18) we will limit our analysis to the backlogging case.

\section{The Queue Length Capacitated Facility Location Problem}

\subsection{Model formulation}

The queue length capacitated facility location problem (QLCFLP) explicitly constrains the capacity of each facility to a given queuing policy. Imposing a limit $\bar{\rho}$ to the backlogging probability at each of the facilities results in the following formulation: 
$\min \sum_{\mathrm{i}=1}^{\mathrm{m}} \sum_{j=1}^{n} C_{i j} X_{i j}+\sum_{j=1}^{m} f_{j} Y_{j}$

s.t.

$\rho_{\mathrm{j}}^{Z} \leq \bar{\rho}$

$\forall \mathrm{j}$

$\sum_{j=1}^{m} X_{i j}=1$

$\forall \mathrm{i}$

$X_{i j}-Y_{j} \leq 0$

$\forall \mathrm{i}, \forall \mathrm{j}$

$\mathrm{X}_{\mathrm{ij}} \in\{0,1\}$

$\forall \mathrm{i}, \forall \mathrm{j}$

$\mathrm{Y}_{\mathrm{j}} \in\{0,1\}$

$\forall \mathrm{j}$

where,

$\rho_{j}=\frac{\sum_{i=1}^{m} \text { freq }_{i} X_{i j}}{\mu}$

is the utilization factor.

Backlogging may be defined as the number of customers in a queue waiting for the product or service. Imposing a limit to the backlogging probability is equivalent to restricting the demand assigned to each facility.

The arrival rate at a facility site $\mathrm{j}$ is defined as the sum of the frequencies of all demand points assigned to this facility. $\mu$ is the service rate. In order to have a stationary system one should add the restriction that the service rate is large enough to cover the arrival rate, i.e.

$\rho_{j}=\frac{\sum_{i=1}^{m} \operatorname{freq}_{i} X_{i j}}{\mu}<1$

Using definition (28) expression (22) can be rewritten as 
$\sum_{i=1}^{n} f_{i} X_{i j} \leq \mu \sqrt[z]{\bar{\rho}}$

which is linear on the decision variables. For a given limit $\bar{\rho}$ and for a given service rate $\mu$ the capacities are defined by $\mathrm{Z}$, the maximum number of items that can be reached.

In comparison with the traditional SSCFLP one would expect a much smaller sensitivity of the solution to variations in the capacity level. It is only for small capacity levels that the impact seems to be relevant. Figure 1 illustrates the case where $\bar{\rho}=0.5$.

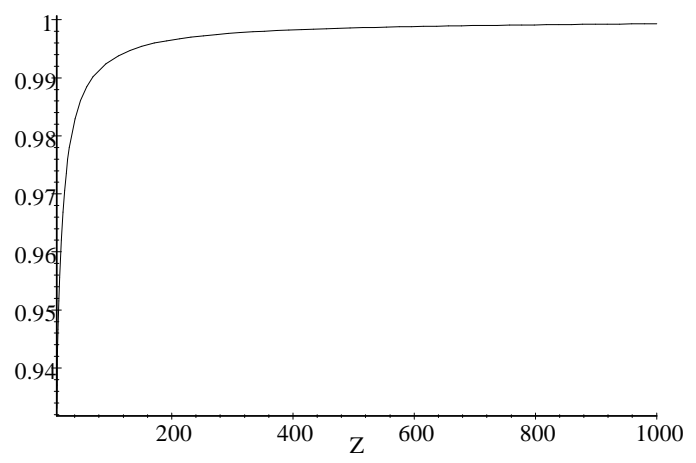

Figure 1: graph of the fuction $f(z)=\sqrt[z]{0.5}$ on the domain 10-1000.

\subsection{Numerical examples}

As a numerical exercise we randomly generated five examples of different size and solved the QLCFLP for three capacity levels (Z). The coordinates of the demand points were generated with a uniform distribution in 50x50 square. Distances between demand points are Euclidean. The population at each demand point was generated from a uniform distribution between 80 and 800 . Demand was fixed at $10 \%$ of the population. Fixed costs were generated from a uniform distribution between 400 and 800 .

Table 1 shows the objective value and best locations for each of the examples for the different capacity levels. As expected we do not find many variations in the results. We give additional information concerning computation time in a Pentium(r ) III processor with $128 \mathrm{MB}$ of RAM. 
Table 1 : some numerical results for the QLCFLP

\begin{tabular}{|c|c|c|c|c|c|c|c|c|c|}
\hline \multicolumn{4}{|c|}{$Z=500$} & \multicolumn{3}{|l|}{$Z=1000$} & \multicolumn{3}{|l|}{$Z=5000$} \\
\hline NL $x$ ND & Objective & Locations & CPU time & Objective & Locations & CPU time & Objective & Locations & CPU time \\
\hline $30 \times 30$ & 1389.35 & $1 ; 7$ & $02 \mathrm{~m} 52 \mathrm{~s}$ & 1389.35 & $1 ; 7$ & $02 \mathrm{~m} 50 \mathrm{~s}$ & 1389.35 & $1 ; 7$ & $02 \mathrm{~m} 52 \mathrm{~s}$ \\
\hline $40 \times 40$ & 1494.62 & $10 ; 27$ & $04 \mathrm{~m} 46 \mathrm{~s}$ & 1494.62 & $10 ; 27$ & $04 \mathrm{~m} 37 \mathrm{~s}$ & 1494.62 & $10 ; 27$ & $04 \mathrm{~m} 30 \mathrm{~s}$ \\
\hline $45 \times 45$ & 1524.44 & $31 ; 40$ & $02 \mathrm{~m} 00 \mathrm{~s}$ & 1524.44 & $31 ; 40$ & $01 \mathrm{~m} 43 \mathrm{~s}$ & 1524.44 & $31 ; 40$ & $02 \mathrm{~m} 32 \mathrm{~s}$ \\
\hline $50 \times 50$ & 1963.77 & $28 ; 30 ; 46$ & $33 \mathrm{~m} 48 \mathrm{~s}$ & 1962.61 & $28 ; 30 ; 46$ & $21 \mathrm{~m} 28 \mathrm{~s}$ & 1962.61 & $28 ; 30 ; 46$ & $49 \mathrm{~m} 17 \mathrm{~s}$ \\
\hline $60 \times 60$ & 1983.26 & $28 ; 39 ; 47$ & $40 \mathrm{~m} 15 \mathrm{~s}$ & 1983.26 & $28 ; 39 ; 47$ & $19 \mathrm{~m} 19 \mathrm{~s}$ & 1982.92 & $28 ; 39 ; 47$ & $20 \mathrm{~m} 07 \mathrm{~s}$ \\
\hline
\end{tabular}

NL : number of potential locations

ND : number of demand nodes

\section{A Heuristic algorithm to solve the QLCFLP}

\subsection{Review of Literature}

The SSCFLP is a combinatorial optimization problem that belongs to the class of NP-hard problems. The traditional approach for solving this problem focuses on obtaining good Lagrangean duals, whose solutions improve the lower bounds provided by LP relaxation. Both capacity and demand constraints have been relaxed, obtaining in the first case uncapacitated facility location subproblems and in the second a number of knapsack problems. Some of those Lagrangean Relaxations can be found in (Barceló et al. 1984, Beasley 1988 or Barceló et al. 1991).

As suggested by Krarup and Pruzan (1983) the FLP is a "hard nut to crack, or, to use a more precise characterization, that it is highly unlikely that an exact polynomial time bounded algorithm can ever be devised for its solution". These same authors carachterize the problem in terms of computational complexity, to demonstrate that indeed it belongs to the class of combinatorial optimization problems termed NP-hard.

Several heuristics have been developed for the CFLP. Jacobsen (Jacobsen 1983) generalizes heuristics for the Uncapacitated Plant Location Problem to the capacitated case. The heuristics are ADD, DROP, SHIFT, ALA (alternative location-allocation) and VSM (vertex substituting method). The ADD and DROP procedures are greedy heuristics, where in the first case a facility is added at each of the iterations and in the 
second case a facility is dropped. The chosen facility is always the one where the largest saving on costs is obtained. Both methods are considered construction methods since no revision on early decisions is allowed. More sophisticated heuristics are based on the idea of improving on a known solution. A good example is the Teitz and Bart ( Teitz et al.1968) Vertex Substitution Method.

Cornuejols (Cornuejols et al. 1991) compare several relaxations for the CFLP with classical greedy or interchange heuristics. The authors compute various lower bounds on the objective value relaxing subsets of constraints either completely or in a Lagrangean fashion. The subsets of constraints considered are: demand constraints, capacity constraints, non-negativity and integrality constraints. Based on their experiments the authors suggest the use of a Lagrangean heuristic to solve large instances of CFLP.

Beasley (Beasley 1993) presents a framework for developing Lagrangean heuristics with respect to the location problems: p-median, uncapacitated warehouse location and capacitated warehouse location with or without single source constraints. The author concludes that the heuristics presented in the paper for the four location problems is able to generate optimal or near optimal solutions at reasonable computing cost.

Concerning the SSCFLP, (Delmaire et al. 1999), propose a Reactive GRASP heuristic, a Tabu Search Heuristic, and two different hybrid approaches that combine elements of the GRASP and the Tabu Search methodologies.

Holmberg (Holmberg et al. 1999) propose an exact algorithm for the capacitated facility location problem with single sourcing. Their procedure is based on Lagrangian heuristics using subgradient optimization. The authors combine a strong 
dual approach (the Lagrangian dual) with a strong primal (the repeated matching heuristic).

Cortinhal and Captivo (Cortinhal et al. 2003) use a Lagrangean relaxation to obtain lower bounds for the SSCPLP, and Lagrangean heuristics followed by search methods and one Tabu Search metaheuristic to obtain upper bounds. The same authors, (Cortinhal et al. 2004), use genetic algorithms to solve the SSCPLP.

\subsection{Heuristics}

Solving the CPLP comprises two sub-problems: finding the optimal location of the facilities and the assignation of demand points to each one of the open facilities. In fact, for any vector $\bar{Y}$ of location variables the optimal solution for the flow variables $\mathrm{X}(\overline{\mathrm{Y}})$ can be retrieved by solving the associated transportation problem:

$\min Z(\bar{Y})=\sum_{i=1}^{m} \sum_{j=1}^{n} C_{i j} X_{i j}$

s.t.

$\rho_{j}{ }^{Z} \leq \bar{\rho}$

$\sum_{j=1}^{m} X_{i j}=1 \quad \forall i$

$X_{i j}-\bar{Y}_{j} \leq 0 \quad \forall i, \forall j$

$X_{i j} \in\{0,1\} \quad \forall i, \forall j$

In the heuristic procedure we used a Reactive-GRASP algorithm and two different types of neighbourhood search: shift neighbourhood and swap neighbourhood.

Reactive GRASP, proposed by Prais and Ribeiro (Prais et al. 2000), is a procedure in which the parameter is self-adjusted according to the quality of the solutions previously found. Instead of fixing the value of the parameter $\gamma$, which determines which elements will be placed in the restricted candidate list, R-GRASP 
randomly selects this parameter value from a discrete set $\left\{\gamma_{1}, \ldots, \gamma_{\mathrm{m}}\right\}$. The probability distribution used in the $\gamma$ selection will be updated after the execution of each block of iterations considering the quality of the solutions obtained by each of the $\gamma_{i}$.

Let $\varphi$ be the greedy function for a minimization problem. The Restricted Candidate List (RCL) contains all the candidate solutions within a given distance of the top candidate as a function of $\varphi$. The threshold value can be expressed as:

$$
\gamma\left(\varphi_{\max }-\varphi_{\min }\right)
$$

The Reactive GRASP selects the best value of $\gamma$, by measuring the goodness of each possible value $\gamma$ and defining an automated selection criterion for this parameter's value at the different iterations of the process.

The algorithm we used to solve our problem comprises the following steps:

1- Set initial probabilities $P_{i}=\frac{1}{v}$ with $i=1, \ldots, v$. $P_{i}$ is the probability of choosing a given parameter $\gamma=\gamma_{i}$. V is the number of candidates for $\gamma$. In our particular case we considered $\mathrm{v}=10$ and a set of candidates $\{0.1, \ldots, 1\}$

2- For each of the blocks of iterations $k=1, \ldots, n u m \_b l o c k s$, repeat the following steps:

2.1- For a given number of iterations $r=1, \ldots$, num_iterations repeat:

2.1.1- Randomly select $\gamma=\gamma_{i}$ from $\left\{\gamma_{1}, \ldots, \gamma_{v}\right\}$ using probabilities $P_{i}$ with $i=1, \ldots, v$.

2.1.2- Construction phase: construct a greedy randomized solution, considering the selected value of $\gamma$.

2.1.4- Apply local search.

2.2.- Update $\gamma$ 's utility ut $(\gamma)$. We considered the utility of $\gamma$ as given by the average deviation of the objectives found using this particular $\gamma$ from the best value for the objective found so far. 
2.3. Compute new probabilities Pi using the following expression: $P_{i}=\frac{u t\left(\gamma_{i}\right)}{\sum_{j=1}^{v} u t\left(\gamma_{j}\right)}$

2.4. Go back to step 2.1 and start a new block of iterations.

The greedy function used in step 2.1.2 was formulated as

$$
\varphi_{j}=\left(f_{j}+\sum_{i \in C_{j}} \Delta_{i j}\right) /\left|C_{j}\right|
$$

where, $\Delta \mathrm{ij}$ is the increment in the objective value for serving client $\mathrm{i}$ from plant $\mathrm{j}$ and $\mathrm{Cj}$ is the set of all unassigned clients that fit into location $\mathrm{j}$. At each step of the construction phase one plant is opened and several clients are assigned to it. The capacity constraints are never violated by partial solutions. Within each plant, clients are ordered by increasing values of the $\Delta \mathrm{ij}$.

In the local search phase we used the well known Teizt and Bart algorithm.

For each group of facility locations the following sub-algorithm was implemented to solve the corresponding transportation problem.

1. Construct an initial solution: assign each demand point to its closest facility location.

2. Explore Shift neighbourhood: starting with the first demand point in your list change its actual allocation by all other possible allocations, one at a time and compute the respective transportation problem's objective. If the solution improves (lower objective) keep the new allocation, otherwise restore the initial allocation. Repeat the procedure for all demand points in the list.

3. Explore Swap neighbourhood: starting with the first demand point in your list swap its actual allocation with all other demand point's allocations, one at a time computing the respective transportation problem's objective. If the 
solution improves (lower objective) keep the new allocations, otherwise restore the initial allocations. Repeat the procedure for all demand points in the list.

We explore both neighbourhoods for all demand points and repeat the searching process while there are improvements to the solution.

In the Capacitated Facility Location Problem there is no a priori information about the number of facilities to be located. In our algorithm we started with one facility and applied an algorithm, which increases the number of facilities by one unit at each block of iterations. The algorithm stops when there are no improvements in the objective by locating one extra facility.

Some other authors apply a neighbourhood search, which allows opening or closing facilities; see as an example (Delmaire et al. 1999).

\subsection{Numerical examples}

In order to evaluate the heuristics we randomly generated 30 examples using the same procedure described in section 4.2. Each of the examples was solved for the optimal using the LINGO commercial package and the heuristics described in the previous points. Table 2 shows the results regarding the experiments.

As shown in table 2, the results are quite close: from the twenty examples we didn't reach the minimum objective in five of the examples. The heuristics allow some important savings in computing times even for small examples. 
Table 2: Heuristics statistics.

\begin{tabular}{|c|c|c|c|c|c|c|}
\hline \multirow[b]{2}{*}{ EXAMPLE } & \multicolumn{3}{|c|}{ LINGO } & \multicolumn{3}{|c|}{ HEURISTICS (10 bocks 10 iter) } \\
\hline & Objective & Locations & $\begin{array}{c}\text { CPU time } \\
(\mathrm{sec})\end{array}$ & Objective & Locations & $\begin{array}{c}\text { CPU time } \\
(\mathrm{sec})\end{array}$ \\
\hline 1 & 1275,64 & $5 ; 28$ & 127,2 & 1275,64 & $5 ; 28$ & 26,42 \\
\hline 2 & 1376,32 & $3 ; 11$ & 154,8 & 1376,32 & $3 ; 11$ & 29,01 \\
\hline 3 & 1308,87 & $5 ; 24$ & 139,2 & 1308,87 & $5 ; 24$ & 24,66 \\
\hline 4 & 1244,58 & $7 ; 12$ & 70,8 & 1244,58 & $7 ; 12$ & 44,71 \\
\hline 5 & 1439,62 & $7 ; 30$ & 138,6 & 1439,62 & $7 ; 30$ & 22,85 \\
\hline 6 & 1395,58 & $2 ; 24$ & 137,4 & 1395,58 & $2 ; 24$ & 63,55 \\
\hline 7 & 1338,24 & $8 ; 10$ & 136,8 & 1338,24 & $8 ; 10$ & 64,76 \\
\hline 8 & 1313,01 & $19 ; 25$ & 83,4 & 1351,75 & -- & 29,01 \\
\hline 9 & 1324,72 & $7 ; 22$ & 144,6 & 1325,59 & -- & -- \\
\hline 10 & 1336,51 & $29 ; 30$ & 133,2 & 1386,94 & -- & -- \\
\hline 11 & 1409,55 & $1 ; 8$ & 264 & 1409,55 & $1 ; 8$ & 33,59 \\
\hline 12 & 1275,83 & $4 ; 22$ & 126 & 1290,56 & -- & -- \\
\hline 13 & 1298,87 & $10 ; 25$ & 183,6 & 1298,87 & $10 ; 25$ & 61,96 \\
\hline 14 & 1287,75 & $19 ; 29$ & 252,6 & 1287,75 & $19 ; 29$ & 23,13 \\
\hline 15 & 1243,1 & $13 ; 22$ & 210 & 1243,1 & $13 ; 22$ & 24,33 \\
\hline 16 & 1279,65 & $2 ; 11$ & 190,2 & 1279,65 & $2 ; 11$ & 21,2 \\
\hline 17 & 1370,32 & $8 ; 28$ & 154,2 & 1370,32 & $8 ; 28$ & 44,87 \\
\hline 18 & 1363,04 & $6 ; 21$ & 187,2 & 1363,04 & $6 ; 21$ & 39,22 \\
\hline 19 & 1279 & $6 ; 28$ & 211,8 & 1279 & $6 ; 28$ & 38,45 \\
\hline 20 & 1250,29 & $20 ; 26$ & 191,4 & 1250,29 & $20 ; 26$ & 24,17 \\
\hline 21 & 1355,67 & $7 ; 30$ & 82,8 & 1355,67 & $7 ; 30$ & 44,05 \\
\hline 22 & 1270,33 & $24 ; 28$ & 144 & 1270,33 & $24 ; 28$ & 24,88 \\
\hline 23 & 1234,12 & $18 ; 27$ & 244,8 & 1234,12 & $18 ; 27$ & 24,39 \\
\hline 24 & 1336,9 & $2 ; 26$ & 129 & 1336,9 & $2 ; 26$ & 33,06 \\
\hline 25 & 1354,75 & $16 ; 20$ & 249 & 1354,75 & $16 ; 20$ & 23,73 \\
\hline 26 & 1302,29 & $16 ; 30$ & 191,4 & 1302,29 & $16 ; 30$ & 26,64 \\
\hline 27 & 1260,3 & $4 ; 15$ & 144,6 & 1292,43 & -- & -- \\
\hline 28 & 1296,69 & $7 ; 23$ & 154,2 & 1296,69 & $7 ; 23$ & 22,58 \\
\hline 29 & 1247,54 & $3 ; 7$ & 73,2 & 1247,54 & $3 ; 7$ & 34 \\
\hline 30 & 1254,24 & $15 ; 19$ & 144,6 & 1254,24 & $15 ; 19$ & 24,33 \\
\hline \multicolumn{4}{|c|}{ Number of distinct solutions (\%) } & & $16 \%$ & \\
\hline \multicolumn{3}{|c|}{ Average deviation (\%) } & & & $0.34 \%$ & \\
\hline \multicolumn{3}{|c|}{ Maximum deviation (\%) } & & & $4 \%$ & \\
\hline \multicolumn{3}{|c|}{ Average CPU time - LINGO } & & \multicolumn{3}{|c|}{$259.82 \mathrm{~s}$} \\
\hline \multicolumn{3}{|c|}{ Average CPU time - Heuristics } & & \multicolumn{3}{|c|}{$33.59 \mathrm{~s}$} \\
\hline
\end{tabular}




\section{A simulation exercise}

Since stochastic systems in general are easy to simulate and an objective function can be computed for each of the simulated scenarios, simulation can be combined with optimization algorithms in order to optimize many real life problems. For a good presentation of the role of simulation in optimization techniques, refer to the textbook by (Gasovi 2003).

One of the assumptions of the model formulated in previous sections consists of observing a stochastic demand whose arrival rate follows a Poisson distribution. A simple exercise developed in this section consists of simulating one arrival process at each one of the demand points for one hundred simulations. Then, we solved the QLCFLP and check if the solution changes at each one of the simulations. We used the heuristic described in the previous section to solve each of the problems.

Across the different examples we maintain the distance matrix, as well as the location of the demand points. The only parameter changing is the arrival rate at each of the demand points. These rates were simulated from a Poisson distribution with a specific parameter (average) for each demand point. For simplicity only we considered twenty demand points. The average arrival rate at each of the demand points is shown in the following table:

Table 3: A Simulation Exercise: Average arrival rate.

\begin{tabular}{ccccccccccccccccccccc}
\hline $\boldsymbol{i}$ & 1 & 2 & 3 & 4 & 5 & 6 & 7 & 8 & 9 & 10 & 11 & 12 & 13 & 14 & 15 & 16 & 17 & 18 & 19 & 20 \\
\hline $\boldsymbol{\lambda}$ & 72 & 40 & 30 & 68 & 19 & 24 & 15 & 75 & 48 & 71 & 47 & 25 & 72 & 56 & 77 & 37 & 66 & 50 & 78 & 47 \\
\hline
\end{tabular}

If we consider, as an example a service rate of two hundred $(\mu=200)$ and a maximum of two hundred tags available $(Z=200)$, the left hand side of equation .... for different capacity levels, measured in terms of utilization ratio $(\bar{\rho})$ are the ones in table 4: 
Table 4: A Simulation Exercise: Capacities.

\begin{tabular}{cc}
\hline $\bar{\rho}$ & LHS \\
\hline 0,1 & 197,7106 \\
0,2 & 198,397 \\
0,3 & 198,7996 \\
0,4 & 199,0858 \\
0,5 & 199,3081 \\
0,6 & 199,4898 \\
0,7 & 199,6436 \\
0,8 & 199,777 \\
0,9 & 199,8947 \\
\hline
\end{tabular}

We run the simulations for the extreme cases and compare the objectives for the extreme cases, where $\bar{\rho}=0,1$ and $\bar{\rho}=0,9$. The results are shown in figure $2 \mathrm{a}$ ) and in figures $2 b$ ) respectively.

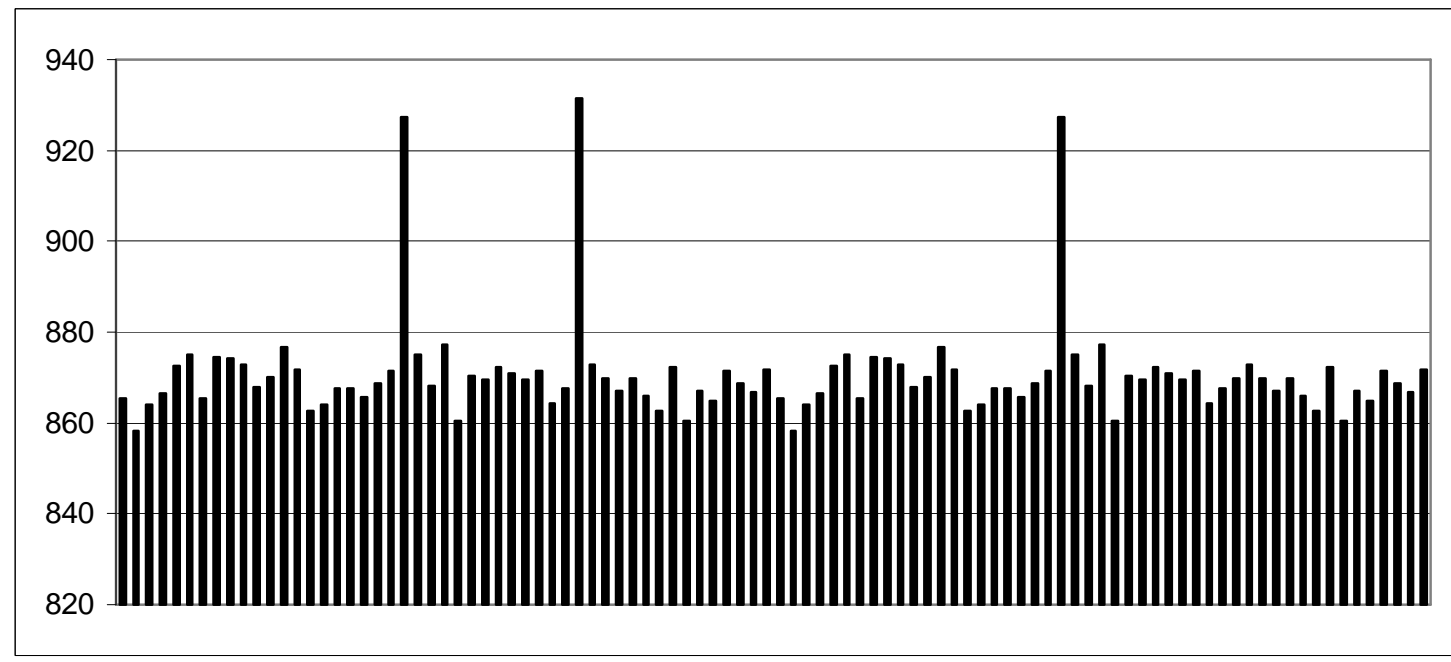

Average Objective 870,5968 Standard Deviation 11,20544

Figure 2a): Objective values : $\bar{\rho}=\mathbf{0 , 9}$ 


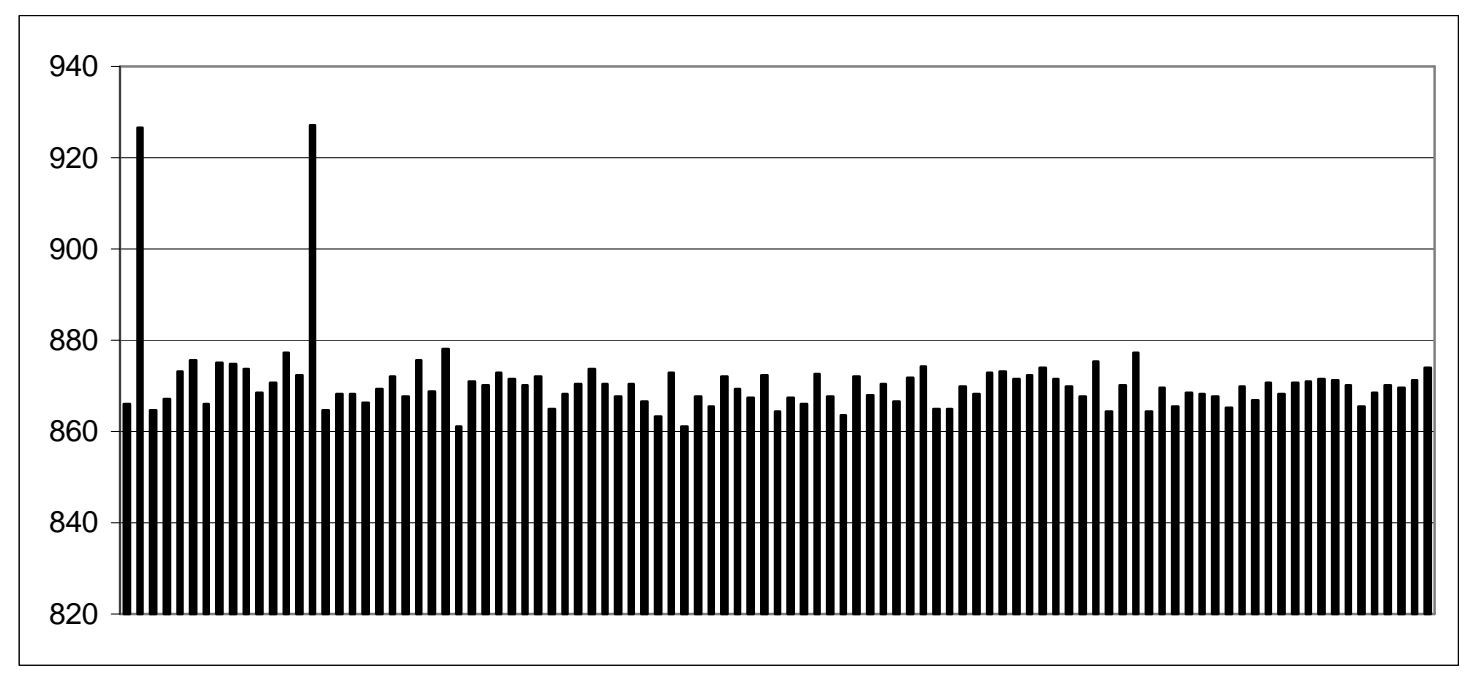

Average Objective

870,7439

Standard Deviation 8,857857

Figure 2b): Objective values : $\bar{\rho}=\mathbf{0 , 1}$

The resulting graphs suggest that in spite of in some exceptional cases the objectives may be different; the average objective is quite similar when we change the upper limit for the utilization ratio. The standard deviations in both cases are relatively low.

\section{Conclusions}

This paper considers a new formulation for the Single Source Capacitated Facility Location Problem in which capacity constraints result from imposing an upper bound to the probability of customers' demand being backlogged. Demand is assumed to be stochastic, following a Poisson distribution and coincides with the arrival rate of a Markovian M/M/1 queuing process.

Theory on stochastic manufacturing systems as well as some numerical examples suggests that solutions in this new model become less sensitive to variations in capacities. 
Knowing the probability distribution of the demand, it is possible to simulate demand. Some simulated examples show that the results do not vary much across the different scenarios.

Finally, greedy heuristics seems to behave well when solving this new formulation of the Single Source Capacitated Facility Location Problem. 


\section{References}

Balinski, M. L. Integer Programming: Methods, uses, computations. Management Science, 1965, 12, 253-313.

Balinski, M. L. On finding integer solutions to linear programs. Proc. IBM Scientific Symposium on Combinatorial Problems, 1966. 225-248.

Balinski, M. L. and K. Spielberg. Methods for integer programming: algebraic, combinatorial, and enumerative. Progress in Operations Research, 1969. 195-292.

Barceló, J. and J. Casanovas. A heuristis algorithm for the Capacitaed Plant Location Problem European Journal of Operational Research, 1984, 15(2),212-226.

Barceló, J. E. Fernandéz and K. Jörnsten. Computational results from a new Lagrangean Relaxation algorithm for the Capacitated Plant Location Problem. European Journal of Operational Research,1991, 53, 38-45.

Beasley, J. E.. An algorithm for solving large capacitated warehouse location problems. European Journal of Operational Research , 1988, 33, 314-325.

Beasley, J. E. Lagrangean heuristics for location problems. European Journal of Operational Research, 1993, 65, 383-399.

Buzacott, J. and J. Shanthikumar. Stochastic Models of Manufacturing Systems. Prentice Hall Publishers. 1993.

Cornuejols, G., R. Sridharan and J.M. Thizy. A comparison of heuristics and relaxations for the Capacitated Plant Location Problem. European Journal of Operational Research, 1991, 50, 280-297.

Cornuejols, G. Analysis of algorithms for a class of location problems. Technical Report no. 382, SORIE, Cornell University. 
Cortinhal, J. and M. E. Captivo. Upper and lower bounds for the single source capacitated location problem. European Journal of Operational Research, 2003, 151, 333-351.

Cortinhal, J. and M. E. Captivo.Genetic algorithms for the single source capacitated location problem. in Metaheuristics Computer Decision-Making. Edited by Mauricio Resende e Jorge Pinho de Sousa. Kluwer Academic Publishers.2004. pp 187-216.

Delmaire, H., J. A. Díaz and E. Fernández. Reactive GRASP and Tabu search based heuristics for the Single Source Capacitated Plant Location Problem. INFOR, 1999, 37, no. 3 .

Gasovi, A. Simulation-based optimization: an overview. Kluwer Academic Publishers.2003.

Guignard, M. and K. Spielberg. Algorithms for exploiting the structure of the simple plant location problem. Annals of Discrete Math, 1977. 1, 247-228.

Holmberg, K. Exact solution methods for uncapacitated location problems with convex transportation costs. European Journal of Operational Research, 1999, 114. 127140.

Jacobsen, S. K. Heuristics for the capacitated plant location model. European Journal of Operational Research, 1983, 12, 253-261.

Krarup, J. and M. Pruzan. The simple plant location problem: Survey and synthesis. European Journal of Operational Research, 1983, 12. 36-81.

Kuehn, A.A. and M.J. Hamburger. A heuristic program for locating warehouses. Management Science, 1963, 9, 643-666.

Manne, A. S. Plant location under economies-of-scale- decentralization and computation. Management Science, 1964, 11, 213-235. 
Prais, M. and C.C. Ribeiro. Reactive GRASP an application to a Matrix Decomposition Problem in TDMA traffic assignment. INFORMS Journal on Computing, 2000,12, vol.3.

ReVelle, C.S., D. Marks and J.C. Liebman. An analysis of private and public sector location models. Management Science, 1970. 16, 692-707.

Rönnqvist, M., S. Tragantalerngsak and J, Holt. A repeated matching heuristic for the single-source capacitated facility location problem. European Journal of Operational Research, 1999, 116, 51-68.

Suri, R., J. Sanders and M. Kamath. Performance Evaluation of Production Networks in Logistics of Production and Inventory edited by S. Graves, A. Kan and P. Zipkin. Elsevier Science Publishers, 1993, 199-274.

Sridharan R. The capacitated plant location problem. European Journal of Operational Research, 1995, 87, 203-213.

Stecke, K. and J. Aronson. Review of operator/machine interference models. International Journal of Production Research, 1985, 23, 129-151.

Teizt, M.B. and Bart, P. Heuristic methods for estimating the generalized vertex median of weighted graph. Operations Research, 1968, 16(5), 955-961. 


\section{Acknowledgments}

This research has been possible thanks to the grant SFRH/BD/2916/2000 from the Ministério da Ciência e da Tecnologia, Fundação para a Ciência e a Tecnologia of the Portuguese government., and grant SEC2003-1991 from the Ministry of Education and Science, Spain. 